\title{
PARITY, FREE KNOTS, GROUPS, AND INVARIANTS OF FINITE TYPE
}

\author{
V. O. MANTUROV
}

\begin{abstract}
In this paper, on the basis of the notion of parity introduced recently by the author, for each positive integer $m$ we construct invariants of long virtual knots with values in some simply defined group $\mathcal{G}_{m}$; conjugacy classes of this group play a role as invariants of compact virtual knots. By construction, each of the invariants is unaltered by the move of virtualization. Factorization of the group algebra of the corresponding group leads to invariants of finite order of (long) virtual knots that do not change under virtualization.

The central notion used in the construction of the invariants is parity: the crossings of diagrams of free knots is endowed with an additional structure - each crossing is declared to be even or odd, where even crossings behave regularly under Reidemeister moves.
\end{abstract}

\section{Introduction. Basic CONCEPts}

The theory of virtual knots was devised by Kauffman [11; virtual knots correspond to knots in thickened surfaces $S_{g} \times \mathbb{R}$ considered to within isotopies and stabilizations.

The theory of invariants of finite order for classical knots was devised independently by Vassiliev [22] and Gusarov [10. It turned out that many known invariants are expressed in terms of invariants of finite order [3]. A breakthrough in the theory of Vassiliev invariants of finite order for classical knots was marked by the work of Kontsevich [12] in which the structure of the space of Vassiliev invariants was described. Vassiliev invariants of classical knots appeared as the starting point for the work of Goussarov, Polyak and Viro [9] in which the definition of a virtual knot was given; this is equivalent to Kauffman's original definition, which effected the classification of invariants of finite order for classical knots and gives the definition of invariants of finite order for virtual knots. The definition of invariants of finite order for virtual knots given in [9] is very narrow but easy to understand. Here we shall use the notion of Vassiliev invariants of virtual knots according to Kauffman [1].

In the case of knots with thickened surfaces the analogue of Kontsevich's theorem was obtained in [2], but the solution (explicit universal formula) did not seem all that elegant by comparison with the classical case and seemed very remote from practical applications.

Virtual knots are considerably more complicated than classical knots: all the zeroorder invariants of classical knots are constants, whereas the space of zero-order invariants of virtual knots is infinite-dimensional. In fact, there exist infinitely many different types of virtual knots that cannot be converted one into the other by isotopies and change of underpass to overpass; for each such type there is a zero-order invariant taking the value 1 on knots of this type and 0 on knots of other types. Consequently, equivalence classes

2010 Mathematics Subject Classification. Primary 57M25, 57M27.

Key words and phrases. Knot, virtual knot, free knot, invariant, parity, group, invariant of finite order. 
of virtual knots with respect to the operation of changing underpass to overpass, called flat virtual knots, are important for understanding the structure of Vassiliev invariants.

An important simplification of the notion of a flat virtual knot is that of a free knot. Free knots were introduced by Turaev [21] under the name of homotopy classes of Gaussian words. In this same paper he put forward the conjecture that free knots are trivial; this was first refuted by the author in [14] and later by Gibson in [7.

In refuting Turaev's conjecture in [14] an important role was played by the concept of parity: all crossings of a free knot are naturally divided into two types - even and odd.

In particular, the following theorem was proved: Any diagram in which all crossings are odd and to which the second diminishing Reidemeister move cannot be applied is minimal.

In the paper [19] invariants of free knots were constructed with values in the groups $G_{m}$ (see the definition below) which led to simply defined infinite series of finite-order invariants for (long) virtual knots. The groups $G_{m}$ are defined by generators and relations and have simple Cayley graphs; the group $G_{1}$ is isomorphic to the infinite dihedral group. We need this group to construct the invariants of free knots (see below); for the invariants of virtual knots we use richer groups.

By a chord (or Gaussian) diagram we mean the embedding $\left(S^{0} \sqcup \cdots \sqcup S^{0} \subset S^{1}\right)$ consisting of an oriented circle $S^{1}$ and a set of $n$ unordered pairs of points embedded in the circle (all $2 n$ points being pairwise distinct). The pairs of points (in $S^{0}$ ) are called chords of the chord diagram (and we depict each pair in the plane by the chord joining these two points), while the points themselves of each pair are called the ends of the chord. By arcs of the chord diagram we mean the connected components of the complement of the set of all the points. In drawing a circle in the plane we assume that the orientation is counterclockwise.

We say that a chord diagram is marked if an additional point is marked on its circle $S^{1}$, different from the ends of the chord. We consider chord diagrams to within natural equivalence. We say the chords $a$ and $b$ of a (marked or unmarked) chord diagram are linked if the two ends of the chord $b$ lie in different connected components of the complement of the ends of the chord $a$ on the circle $S^{1}$; otherwise, we say that the chords are unlinked; by definition we consider a chord to be unlinked with itself. We say that a chord of a chord diagram is even if the number of chords that are linked with it is even; otherwise, we say that the chord is odd.

We say that a tetravalent graph is equipped if at each of its vertices a partition of the four half-edges incident to it into two pairs is indicated; two half-edges of one pair are called (formally) opposite. The number of unicursal components is defined as the number of equivalence classes of edges, where equivalence is induced by the oppositeness relation in the crossing. Chord diagrams (with a nonempty set of chords) are in oneto-one correspondence with (oriented) equipped tetravalent graphs with one unicursal component.

Each (nonempty) chord diagram defines a certain abstract equipped tetravalent graph that is obtained from the circle of the chord diagram by identifying pairs of points belonging to one chord. At each such vertex two arcs of the circle incident to the same end form formally opposite half-edges of the equipped tetravalent graph.

Corresponding to the empty chord diagram is a circle that is not a graph.

An orientation of an equipped tetravalent graph corresponding to the orientation of the chord diagram is defined on any of its edges and is extended in natural fashion to the opposite edge and then to the entire graph. In the case of an empty chord diagram, instead of a graph we obtain one circle without vertices; allowing the latter as the only exception, we shall use the term "equipped 4-graph" to mean either an actual equipped 
tetravalent graph or the union of an equipped tetravalent graph with a collection of several circles.

We shall also use the term crossings as applied to knots of a vertex of equipped tetravalent graphs.
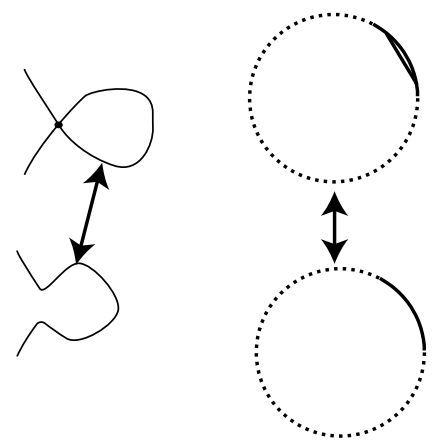

FiguRE 1. The first Reidemeister move and the version using the language of chord diagrams

In what follows we shall be dealing only with equipped tetravalent graphs having one universal component unless otherwise stipulated.

By a free knot [21], 14], we shall mean the equivalence class of chord diagrams with respect to the equivalence generated by three elementary equivalences, called Reidemeister moves (see below). Similarly, by a long free knot we mean an equivalence class of marked chord diagrams under the same Reidemeister moves. In the definition of each of the Reidemeister moves for marked chord diagrams we require that the mark on the chord diagram belong to the unvarying part of the graph (depicted by the dotted line).

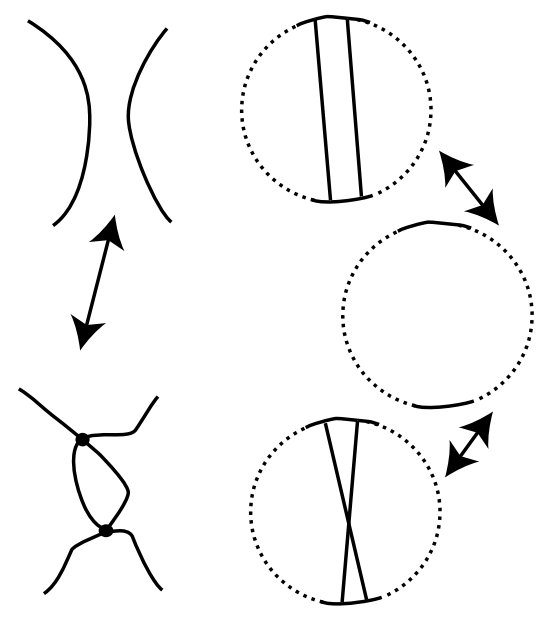

Figure 2. The second Reidemeister move and the version using the language of chord diagrams

Remark 1. Each of the Reidemeister moves consists in a transformation of one fragment of the equipped 4-graph. In the diagram we have merely indicated the rule for this fragment without drawing the remaining part of the diagram. In the case of one unicursal component this corresponds to a transformation on Gaussian diagrams, which 
restructures the diagram on some collection of arcs. In depicting the transformation of the Gaussian diagram we do not show the chords which are not affected by the transformation and we draw the remaining arc using a dotted line.

The first Reidemeister move (Figure1) consists in the addition/removal of a separately standing chord (that is, a chord whose two ends are neighbours). The second Reidemeister move (Figure 2) consists in the removal/addition of two neighbouring chords. Chords $a$ and $b$ are said to be neighbours if their ends $a_{1}, a_{2}$ and $b_{1}, b_{2}$ can be renumbered in such a way that $a_{1}$ and $b_{1}$ are neighbours and $a_{2}$ and $b_{2}$ are neighbours.

The third Reidemeister move is depicted in Figure 3 and consists in the following. Suppose that there are three pairwise linked chords $a, b, c$ whose ends can be reordered in such a way that the end $a_{1}$ of chord $a$ is a neighbour of the end $c_{2}$ of chord $c$, as are the ends $b_{1}$ of chord $b$ and $a_{2}$ of chord $a$, and the ends $c_{1}$ of chord $c$ and $b_{2}$ of chord $b$. The transformed chord diagram will have three new pairwise unlinked chords: $a^{\prime}$ with ends in the positions $c_{2}, b_{1} ; b^{\prime}$ with ends at $a_{2}, c_{1}$; and $c^{\prime}$ with ends at $b_{2}, a_{1}$.

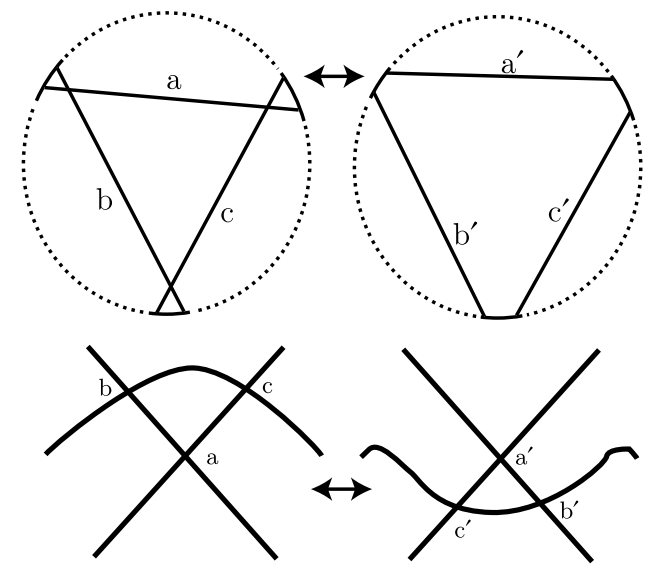

FiguRE 3. The third Reidemeister move and the correspondence of crossings

Remark 2. We note that there is one further similar move, which is also called the third Reidemaister move; it is represented as the composition of the moves depicted in Figure 3 and second Reidemeister moves; thus its addition to the list of Reidemeister moves does not change the concept of the equivalence of chord diagrams.

There is the obvious notion $\mathrm{Cl}$ of the closure of a set of marked chord diagrams in a set of chord diagrams consisting in forgetting the marked point. It is clear that equivalent marked chord diagrams (via Reidemeister moves) go over to equivalent unmarked chord diagrams. Thus the operation which consists in converting long free knots into free knots is well defined. (We shall also call this operation taking the closure and denote it by $C l$.) This operation is not one-to-one; that is, there are inequivalent long free knots corresponding to long free knots.

The Reidemeister moves given above arise naturally from Reidemeister moves for classical and virtual knots.

1.1. The axiomatics of parity. We shall say that a crossing of an equipped 4-graph with one unicursal component is even if the chord of the chord diagram corresponding to it is even. 
Note that the parity of marked or unmarked chord diagrams (or equipped 4-graphs with one unicursal component) defined above (we also call it the Gaussian parity) has the following properties.

(1) If $K_{2}$ is obtained from $K_{1}$ by the first Reidemeister move, then the crossing participating in the Reidemeister move of Diagram $K_{1}$ is even.

(2) If $K_{2}$ is obtained from $K_{1}$ by the second Reidemeister move, then both crossings participating in the Reidemeister move have the same parity.

(3) If $K_{2}$ is obtained from $K_{1}$ by the third Reidemeister move, then there is a natural correspondence between the triple of crossings in Diagram $K_{1}$ and the triple of crossings in Diagram $K_{2}$ which participate in the Reidemeister move $\left(\left(a, a^{\prime}\right),\left(b, b^{\prime}\right),\left(c, c^{\prime}\right)\right)$; see Figure 4 .

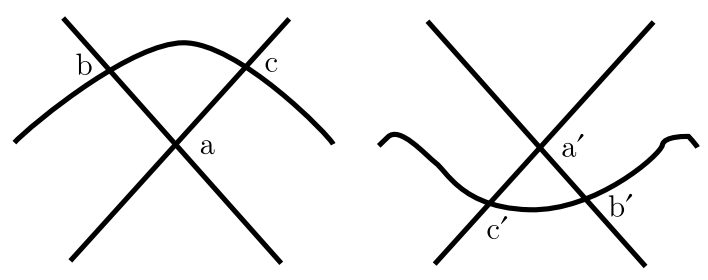

FiguRE 4. The correspondence between the crossings for the third Reidemeister move

Then:

a) the parity of corresponding crossings is the same,

b) the number of odd crossings from the crossings $a, b, c$ is even (that is, it is equal to 0 or 2$)$.

(4) Under each Reidemeister move $K_{1} \rightarrow K_{2}$ there is a one-to-one correspondence between the crossings of Diagram $K_{1}$ not taking part in the Reidemeister move and the crossings of Diagram $K_{2}$ not taking part in the Reidemeister move. Corresponding crossings have the same parity.

The properties of Gaussian parity given above can be taken as the axioms for the definition of parity. In [14, examples are given of various parities for the theory of virtual knots.

For any theory of knots with parity in which the knots are given by equivalence classes of chord diagrams (perhaps with additional structures) with respect to Reidemeister moves (in particular, for the theory of free knots with Gaussian parity) the operation $f$, which removes odd crossings, is well defined. (In the case of chord diagrams, the odd chords are removed.)

Namely, we have the following result.

Theorem 1 ([21], [14]). If the diagrams $D$ and $D^{\prime}$ are equivalent (that is, they give equivalent free knots), then the diagrams $f(D)$ and $f\left(D^{\prime}\right)$ are also equivalent.

The proof of this theorem follows directly from the juxtaposition of the Reidemeister moves with the axioms (parity properties). This was first pointed out for Gaussian parity by Turaev and in the general case by the author in [14.

In what follows we shall need the map $f$ to define the invariants of free knots. Note that the map $f$ is not an idempotent in Gaussian parity: in removing odd crossings, former crossings that were previously even can become odd; see Figure 5 .

We note one further important consequence of the fact that $f$ is well defined. 


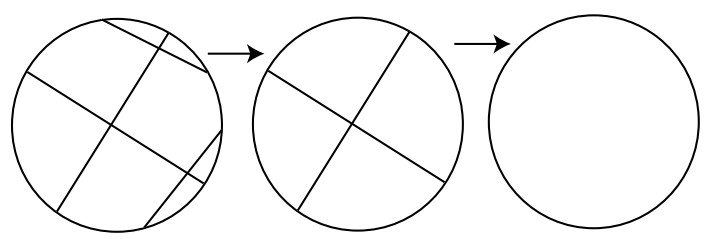

Figure 5. Double application of the operation $f$

Theorem 2 (14]). Let $\mathcal{K}$ be some some theory of knots with parity and let $K_{1}, K_{2}$ be two equivalent diagrams in this theory in which all the crossings are even. Then these diagrams are equivalent by means of a chain of Reidemeister moves in which all the intermediate diagrams are even.

The proof of the theorem consists in successive applications of the map $f$ to transform an arbitrary chain of diagrams with Reidemeister moves into a chain in which all the intermediate diagrams are even.

This means that in the theory of knots with parity there is a natural class of even knots, namely those which have even diagrams; here two even knots are equivalent if and only if they are equivalent by means of moves in the class of even diagrams.

One way of constructing invariants of free knots with the use of the map $f$ is the following. We consider an arbitrary invariant $i$ and choose a composite of the invariant $i$ with the "forgetful" map: $i \circ f$ defines an invariant of the original knot whose value is equal to the value of the invariant $f$ on the modified knot. Here we can partly "remember" the crossings (chords) obliterated by the map $f$. Via this route a sharpening of the Kauffman brackets was constructed in 14, a sharpening of the quandles and Alexander polynomials in [1], and new combinatorial formulae for finite-order invariants in [5].

1.2. Long knots. Construction of invariants. We fix a positive integer $n$. Consider the group

$$
\begin{gathered}
G_{m}=\left\langle a_{0}^{\prime}, a_{0}^{\prime \prime}, \ldots, a_{m-1}^{\prime}, a_{m-1}^{\prime \prime}, a_{m}:\right. \\
\left(a_{m}\right)^{2}=e,\left(a_{i}^{\prime}\right)^{2}=e,\left(a_{i}^{\prime \prime}\right)^{2}=e, i=0, \ldots, m-1, \\
\left.a_{i}^{\prime} a_{j}^{\prime}=a_{j}^{\prime} a_{i}^{\prime \prime}, i<j ; \quad a_{i}^{\prime} a_{j}^{\prime \prime}=a_{j}^{\prime \prime} a_{i}^{\prime \prime}, i<j ; \quad a_{i}^{\prime} a_{m}=a_{m} a_{i}^{\prime \prime}, i=0, \ldots, m-1\right\rangle .
\end{gathered}
$$

With each element of the group $G_{m}$ we associate a marked chord diagram $D$; to do this we associate with each chord its index and type. Recall that the map $f$ associates with a chord diagram the chord diagram obtained by removing the odd chords from it.

We say that in a chord diagram $D$, odd chords have index 0 , chords that are odd chords of the diagram $f(D)$ have index 1 , and so on, by induction; thus chords of index $m-1$ are those chords that are odd in the diagram $f^{m-1}(D)$; the remaining chords (belonging to the diagram $f^{m}(D)$ ) have index $m$. Chords of index $m$ do not have a type; we associate with each chord of index $k<m$ a (first or second) type, depending on whether the number of even chords in the diagram $f^{k}(D)$ linked with the given chord is even or odd. As we move around the circle from the marked point of the diagram $D$ in accordance with its orientation, on encountering an end of the chord with index $i$ of type $u$ (here $u=1$ or $u=2$ ) we write the generator of the group $G_{m}$ with the index $i$ and with $u$ dashes (if the index is $m$, then we write the generator $a_{m}$ ); we denote the word obtained in this way by $\gamma(D)$, and the corresponding element of the group $G_{m}$ by $[\gamma(D)]$.

Figure 6 shows a chord diagram of a free knot and the indices and types of the chords are indicated for $m=2$. 


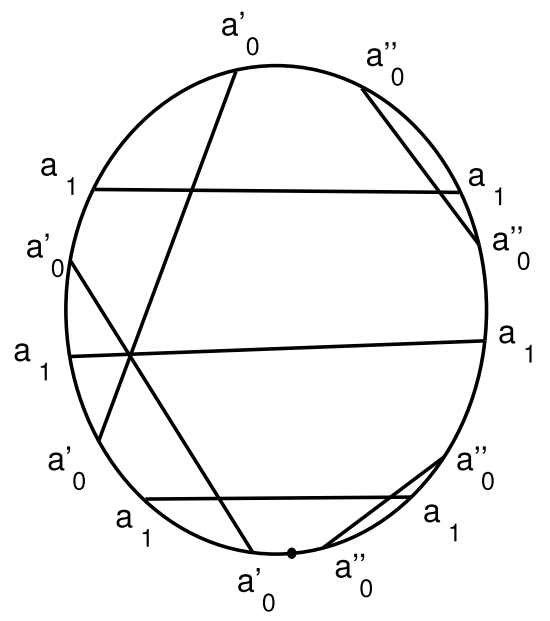

FIgURE 6. A free knot with a nontrivial value of $\gamma$

The following assertions are proved in [19].

Theorem 3. 1. The element $[\gamma(D)]$ is an invariant of long free knots.

2. The conjugacy class of $[\gamma(D)]$ is an invariant of free knots.

Example 1. Consider the long free knot given by the chord diagram depicted in Figure 6. It is easy to verify that (for $m=1$ ) the value of the invariant $[\gamma]$ of this knot is equal to $\left(a_{0}^{\prime \prime} a_{0}^{\prime}\right)^{4}$ and for different choices of the initial point for the closure of this knot the values of the invariant can be both $\left(a_{0}^{\prime \prime} a_{0}^{\prime}\right)^{4}$ and $\left(a_{0}^{\prime \prime} a_{0}^{\prime}\right)^{-4}$. It follows from this that there can be different (inequivalent) long free knots with equivalent closures.

It can be shown that for $m=1$ the value of the invariant $\gamma$ on any long free knot is equal to $\left(a_{0}^{\prime \prime} a_{0}^{\prime}\right)^{4 l}$ for some integer $l$.

The elements of the group $G_{m}$ are in one-to-one correspondence with the integer points in the Euclidean space $\mathbb{R}^{m+1}$ whose last coordinate is equal to zero or one. The identity of the group corresponds to the origin. The process of associating a point in Euclidean space with an element of the group is effected by induction. Suppose that for some element $g \in G_{m}$, given by its word of generators, the point in Euclidean space has already been defined; we define points corresponding to the elements $g \alpha$, where $\alpha$ is a generator of $G_{m}$. Right multiplication by a generator with subscript $k$ ( $a_{k}^{\prime}$ or $a_{k}^{\prime \prime}$ or, in the case $k=m$, the generator $a_{m}$ ) corresponds to a shift of one step in the coordinate with index $k+1$. The direction of the shift is defined in the following manner: for $0 \leqslant k \leqslant m-1$, if the sum of all the coordinates apart from the first $k$ is even, then multiplication by $a_{k}^{\prime}$ means increasing the coordinate with index $k+1$ and multiplication by $a_{k}^{\prime \prime}$ means decreasing this coordinate; if the sum of the coordinates is odd, then $a_{k}^{\prime}$ and $a_{k}^{\prime \prime}$ exchange roles; multiplication by $a_{m}$ corresponds to changing the last, $(m+1)$ st coordinate (from zero to one or one to zero). It can be verified directly that this correspondence is well defined; that is, it does not depend on how the element of the group is written in the form of a product of generators of the group.

It is not difficult to verify that corresponding to a chord diagram there is an element of the group whose last coordinate equals zero.

We now describe how elements of a conjugacy class in the group $G_{m}$ behave.

The following assertion can be verified directly. 
Proposition 1. 1) If an element $p \in G_{m}$ is realized in the form of an invariant of some chord diagram, then all its coordinates are even and the last one is equal to zero.

2) Two elements $p$ and $q$ in a group $G_{m}$ all of whose coordinates are even and whose last coordinate is equal to zero are conjugate if and only if for each $j$ their $j$ th coordinates $p_{j}$ and $q_{j}$ have the same absolute value: $\left|p_{j}\right|=\left|q_{j}\right|$.

Proof. The proof of part 1) is obvious. The proof of part 2) is a consequence of the following argument: we represent each element of the indicated form as a product of positive and negative powers of elements of the form $u_{k}=\left(a_{k}^{\prime} a_{k}^{\prime \prime}\right)$ : the element $u_{k}$ commutes with the generators of $G_{m}$ with indices that are strictly less than $k$; for generators $w$ whose index is greater than or equal to $k$ we have $w u_{k} w^{-1}=u_{k}^{-1}=\left(a_{k}^{\prime \prime} a_{k}^{\prime}\right)$; this proves part 2).

\section{Virtual Knots}

By a virtual diagram we mean an immersion in general position 11 in the plane of an equipped 4-graph such that the image of each vertex indicates which pair of opposite edges forms an overpass and which an underpass (drawn with a broken line); intersections of images of the interiors of different edges are called virtual crossings and are denoted by a small circle, see Figure 7 .

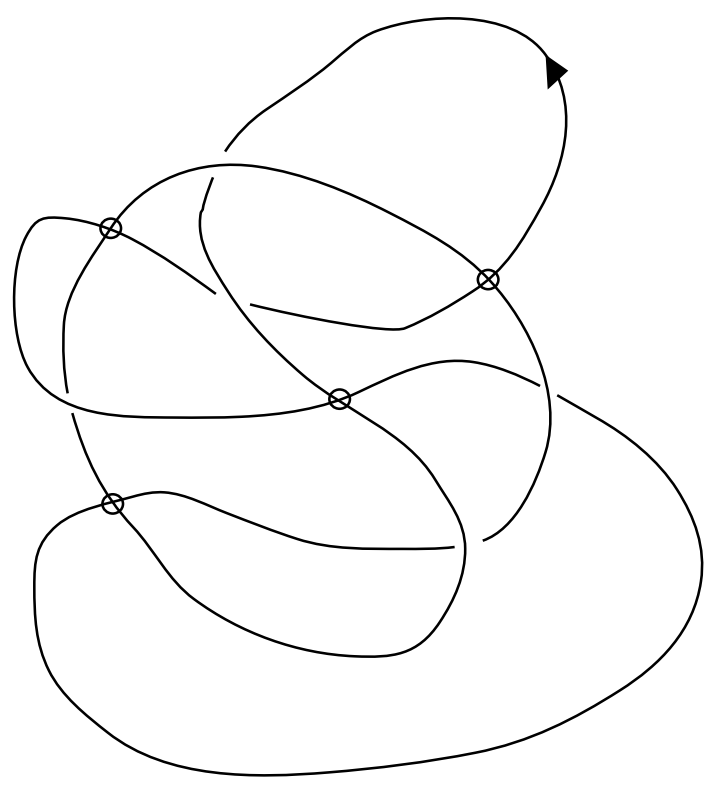

Figure 7. Virtual diagram

By a virtual link we mean the equivalence class of virtual diagrams in terms of the three Reidemeister moves and the 'detour' move (see [16]). The Reidemeister moves are applied to classical crossings. The detour move consists in the removal of an arc containing only

\footnotetext{
${ }^{1} \mathrm{By}$ an immersion in general position of an equipped 4-graph we mean a map of the graph into the plane that is one-to-one at all points of the image apart from finitely many points; at each point of the image where the one-to-one property breaks down we require that there be two inverse images that are internal points of some edges (possibly one and the same edge); it is further required that in the neighbourhood of each double point the intersection of the images of the two branches of the circle be transversal.
} 
virtual crossings and the addition (in an arbitrary fashion) of an immersed arc joining those points which have virtual-crossing signs in the positions with intersections and self-intersections.

The number of unicursal components of a virtual diagram and a virtual link is defined as the number of unicursal components of the corresponding equipped 4-graph; a virtual $k n o t$ is a virtual link of one component. Oriented virtual diagrams and virtual links are defined via an indication of the orientation on each unicursal component. Similarly, associated with (compact) virtual knots we define long virtual diagrams and long virtual knots (see [15]). Instead of introducing a marked point on an edge of the graph (as we did for free knots) we proceed as follows. We consider the "punctured graph" obtained from the equipped 4-graph by removing the marked point. In the immersion in the plane we require that the image of the graph outside some large disc should coincide with the $O x$ axis, and the two connected components of a neighbourhood of the punctured point should be taken to a neighbourhood of $\pm \infty$ and the embedded graph be oriented from $-\infty$ to $+\infty$. We only apply the Reidemeister moves inside some disc (fixed beforehand). This means that a neighbourhood of the punctured point does not participate in the Reidemeister moves.
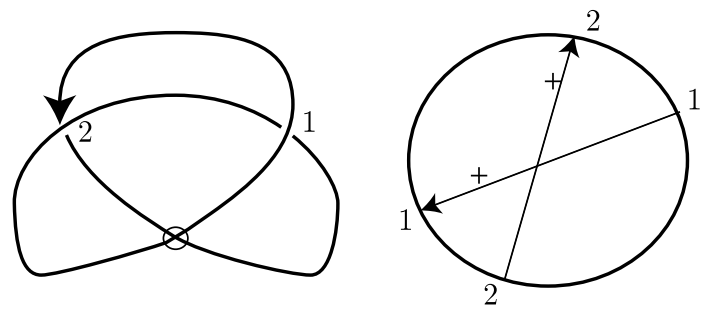

Figure 8. The Gaussian diagram of a virtual knot

Since the diagram of a virtual knot is an immersion of an equipped 4-graph, there is a natural correspondence 9 between it and a chord diagram $\Gamma(K)$. Each chord of this diagram is additionally provided with an arrow and a sign; the arrow on the chord diagram is directed from the inverse image of the arc forming the overpass to the inverse image of the arc forming the underpass; the sign of a crossing of the form $\mathrm{X}$ is positive, while the sign of a crossing of the form $X$ is negative. We associate with a (long) virtual knot the (long) free knot whose chord diagram is obtained from $\Gamma(K)$ by forgetting the arrows and signs. Note that the detour move does not alter the Gaussian diagram and any two virtual diagrams having the same chord diagram are obtained from each other via detour moves. By construction, Reidemeister moves on (long) virtual knots induce the equivalence of chord diagrams of (long) free knots described above, which we also called Reidemeister moves.

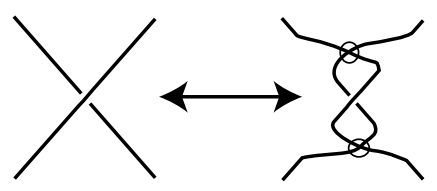

Figure 9. Virtualization

As in the case of free knots, the operation of closure $C l$ is defined on long virtual knots and converts long virtual knots to compact virtual knots. 
By the virtualization transformation of the arrow of a chord diagram we mean changing the direction of this arrow but preserving its sign (and preserving all the directions and signs of all the other arrows). In the language of virtual diagrams, any virtualization transformation looks like the one depicted in Figure 9 to within the detour move. We call this transformation a virtual crossing.

The examination of knots and virtual knots to within virtualization is a natural problem. The point is that the type of a classical knot is completely determined by the chord diagram with signs but without arrows. In the case of virtual knots a natural object arises: chord diagrams without arrows but with signs, considered up to Reidemeister moves. They correspond to virtual knots up to the equivalence induced by the virtualization.

In connection with this the question whether or not this type of equivalence restricts the set of classical knots arises naturally. In other words, this question can be formulated in the form of the following conjecture.

Virtualization conjecture ([6]). Is it true that if two classical diagrams are equivalent via Reidemeister moves and the virtualization transformation, then they give isotopic classical links?

In the case when one of the diagrams defines a trivial knot, the answer to this question is affirmative. This follows from a recent result due to Kronheimer and Mrowka [13, who proved that Khovanov homologies detect the trivial knot in the class of classical knots and also from the construction of the Khovanov homology, which is invariant with respect to virtualization [17. In the general case the question remains open.

Virtual knots to within virtualization occupy an intermediate position between virtual knots and free knots: the subsequent factorization consisting in forgetting the number of twists in crossings leads to free knots.

It is proved in [4] that, in the case of integer coefficients, combinatorial formulae, in the sense of Goussarov-Polyak-Viro do not give invariants that are unaltered under virtualization. The invariants constructed here do not change under virtualization. In the case of coefficients over the field $\mathbb{Z}_{2}$, examples of such invariants were constructed in Gibson's paper [8].

\section{VASSILIEV INVARIANTS}

Let $h$ be an invariant of (long) virtual knots. We say that $h$ is a (Vassiliev) invariant of order at most $k$ if for any virtual knot $K$ with $k+1$ fixed classical crossings we have $\sum_{s}(-1)^{\sharp(s)} h\left(K_{s}\right)=0$, where the sum is taken over all possible $2^{k+1}$ knots $K_{s}$ that coincide with $K$ everywhere except in neighbourhoods of the selected crossings, and at the $k+1$ fixed positions the crossing $X$ or $X$ is chosen arbitrarily, where $\sharp(s)$ denotes the number of crossings of type $X$ among those chosen.

It is clear that invariants of order 0 are those invariants that do not change when an underpass is replaced by an overpass. In particular, all invariants of free knots are of this kind.

Consider the group

$$
\begin{aligned}
& \tilde{G}_{m}=\left\langle a_{0}^{\prime}, a_{0}^{\prime \prime}, \ldots, a_{m-1}^{\prime}, a_{m-1}^{\prime \prime}, a_{m}:\right. a_{m}^{2}=e,\left(a_{i}^{\prime}\right)^{2}=\left(a_{i}^{\prime \prime}\right)^{2}, i=0, \ldots, m-1, \\
& a_{i}^{\prime} a_{j}^{\prime}=a_{j}^{\prime} a_{i}^{\prime \prime}, a_{i}^{\prime} a_{j}^{\prime \prime}=a_{j}^{\prime \prime} a_{i}^{\prime \prime}, a_{j}^{\prime} a_{i}^{\prime}=a_{i}^{\prime \prime} a_{j}^{\prime}, \\
&\left.a_{j}^{\prime \prime} a_{i}^{\prime}=a_{i}^{\prime \prime} a_{j}^{\prime \prime}, i<j ; a_{i}^{\prime} a_{m}=a_{m} a_{i}^{\prime \prime}, i=0, \ldots, m-1\right\rangle .
\end{aligned}
$$

We fix $m$ and write $G=G_{m}$ and also $\tilde{G}=\tilde{G}_{m}$ (in what follows we shall use subscripts to denote the order of Vassiliev invariants). It is easy to show that the group $G$ is obtained from $\tilde{G}$ by adding the relations $\left(a_{i}^{\prime}\right)^{2}=e, i=0, \ldots, m-1$. Let $\mathcal{G}=\mathbb{Q} \tilde{G}$ be 
the group algebra of $\tilde{G}$ and for each $k$ let $\mathcal{G}_{k}$ denote the quotient algebra of $\mathcal{G}$ by the relations $\prod_{j=1}^{k+1}\left(\left(a_{n_{j}}^{\prime}\right)^{2}-1\right)=0$, where $n_{j}$ is an arbitrary subset of numbers from 0 to $m-1$. Clearly, $\mathcal{G}_{0}=\mathbb{Q} G$.

It easily follows from the relations of the group $G$ that any element of $\mathcal{G}_{k}$ has the form $\alpha_{1} A_{1} \cdot \alpha_{2} A_{2} \cdot \ldots \cdot \alpha_{k+1} A_{k+1} \cdot \alpha_{k+2}$ for arbitrary $\alpha$ and $A_{j}=\left(u-u^{-1}\right)$, where $u$ is one of the generators of $G$, is equal to zero.

\section{Construction of neW invariants}

Let $K$ be a long virtual knot. We associate with it an element $\delta(K) \in \tilde{G}$ in the following way. In the Gaussian diagram $\Gamma(K)$ of the knot $K$ we write a word in the alphabet of the generators of $\tilde{G}$ and their inverses in almost exactly the same way as we wrote the word $\gamma(D)$; the only difference is that instead of each generator $a_{j}^{\prime}$ or $a_{j}^{\prime \prime}$, $j=0, \ldots, m-1$, we shall write either that generator or its inverse depending on whether the crossing of the virual knot under consideration is positive $(X)$ or negative $(X)$. We denote the word obtained in this way by $\delta(K)$. Note that, by construction, the word $\delta(K)$ does not depend on the direction of the arrows on the Gaussian diagram of the knot $K$. We denote the element of the group $\tilde{G}$ given by the word $\delta(K)$ by $[\delta(K)]$. We denote the image of the element $[\delta(K)]$ in $\mathcal{G}_{k}$ by $[\delta(K)]_{k}$.

The main result in this paper is the following,

Theorem 4. An element $[\delta(K)]$ of the group $\tilde{G}$ is an invariant of long virtual knots that is not altered by the operation of changing the direction of the arrows in the Gaussian diagram defined by the given knot.

The conjugacy class of an element $[\delta(K)]$ in the group $\tilde{G}$ is an invariant of virtual knots.

For each $k$ the map $K \mapsto[\delta(K)] \in \mathcal{G}_{k}$ is a Vassiliev invariant of long virtual knots of order at most $k$.

Proof. The first assertion of the theorem follows immediately by associating the Reidemeister moves for long virtual knots with relations in the group; by construction, the invariant $[\delta]$ does not depend on the direction of the arrows in the Gaussian diagram; the latter implies it does not alter under virtualization (see [6]). Going over from long virtual knots to compact knots is related to changing the starting point of the bypass; transferring this point through the crossing defines a conjugation of the group $\tilde{G}$; the second assertion of the theorem now follows.

We consider an alternating difference of the values of an invariant $[\delta]$ for long virtual knots corresponding to the arrangement of the $k+1$ crossings of the form $X$ or $X$. By the construction of $[\delta]$, at the position of each difference of crossings there will correspond a factor of one of the forms $\left(a_{i}^{\prime}-\left(a_{i}^{\prime}\right)^{-1}\right)$ or $\left(a_{i}^{\prime \prime}-\left(a_{i}^{\prime \prime}\right)^{-1}\right)$ or $\left(a_{m}-\left(a_{m}\right)^{-1}\right)$; the last factor is equal to zero. Bearing in mind the relation of factorization in $\tilde{G}_{k}$ and the fact that the number of factors in the product is equal to $k+1$, we see that the required alternating difference is equal to zero in $\tilde{G}_{k}$.

4.1. The Cayley graph. The invariants that we have described are constructive. The group $\tilde{G}_{m}$ admits a description that is similar to the description of the group $G_{m}$ given above. The Cayley graph of the group $\tilde{G}_{m}$ is an integer lattice in $\mathbb{R}^{2 m+1}$ with coordinates $x_{1}, \ldots, x_{2 m+1}$, where all the coordinates except the last are arbitrary, while the last coordinate is equal to zero or one; here right multiplication by $a_{m}$ changes the last coordinate, whereas right multiplication by $a_{i}^{\prime}$ or $a_{i}^{\prime \prime}$ signifies a shift in the positive direction for one of the coordinates $x_{2 i+1}$ or $x_{2 i+2}$, and the choice of which coordinate is to be changed depends on the parity of the sum of all the coordinates with indices from $2 i+1$ to $2 m+1$ inclusive. 
The conjugacy classes in the group $\tilde{G}_{m}$ are also easy to describe: under the conjugation of a word with all the coordinates being even and the last coordinate equal to zero the positions of the coordinates with indices $2 i-1$ and $2 i$ for some $i=1,2, \ldots, m$ are exchanged.

4.2. Cobordisms of free knots and further problems. In [18] it was proved that the invariant $[\gamma]$ arising from the group $G_{1}$ mentioned above is an obstruction to the possibility that the knot be cobordant to zero (for the definition, see [18]).

A similar assertion can also be proved for virtual knots by strengthening the structure of the group and introducing new generators. This leads to a certain analogue of the theory of invariants of finite type for cobordisms of virtual knots and also two-dimensional knots in four-dimensional manifolds.

Note that in this paper we only looked at virtual knots to within virtualization; however, by considering virtual knots without factorization one can find more examples of parity (see [14]), update the groups described above significantly (by adding generators corresponding to crossings with a different geometry) and construct new invariants of cobordisms of virtual knots. We will devote a separate paper to this.

\section{ACKNOWLEDGEMENTS}

The author wishes to express gratitude to V. A. Vassiliev and D. P. Il'yutko for a number of valuable comments.

\section{REFERENCES}

[1] D. M. Afanas'ev, Refining virtual knot invariants by means of parity, Mat. Sb. 201 (2010), no. 6, 3-18. Translation: Sb. Math. 201:6 (2010), 785-800. MR2682364 (2011i:57014)

[2] J. E. Andersen and J. Mattes, Configuration space integrals and universal Vassiliev invariants over closed surfaces, Arxiv: q-alg./9704019.

[3] J. Birman and X.-S. Lin, Knot polynomials and Vassiliev's invariants, Invent. Math. 111 (1993), 225-270. MR1198809 (94d:57010)

[4] M. Chrisman, Twist lattices and the Jones-Kauffman polynomial for long virtual knots, J. Knot Theory \& Ramif. 19 (2010), no. 5, 644-675. MR2646652 (2011j:57016)

[5] M. Chrisman and V. O. Manturov, Combinatorial formulae for finite-type invariants via parities, Arxiv.Math/GT.1002.0539.

[6] R. A. Fenn, L. H. Kauffman and V. O. Manturov, Virtual knots: unsolved problems, Fund. Math. 188 (2005), 293-323. MR2191949 (2006k:57011)

[7] A. Gibson, Homotopy invariants of Gauss words, ArXiv: Math.GT/0902.0062. Math. Ann. 349 (2011), no. 4, 871-887. MR2777036

[8] A. Gibson, Finite type invariants of nanowords and nanofrases, ArXiv: Math.GT/1007.1693.

[9] M. Goussarov, M. Polyak and O Viro, Finite type invariants of classical and virtual knots, Topology 39 (2000), 1045-1068. MR1763963(2001i:57017)

[10] M. Gusarov, A new form of the Johns-Conway polynomial for oriented links, Zap. Nauk. Ser. LOMI, no. 193. (Geometry and Topology 1, 4-9). Translation: Adv. Sov. Math. 18, American Mathematical Society, Providence, RI, 1994. MR.1157140(93b:57007)

[11] I. H. Kauffman, Virtual knot theory, Eur. J. Combinatorics 20 (1999), no. 7, 662-690. MR1721925 (2000i:57011)

[12] M. Kontsevich, Vassiliev's knot invariants, Adv. Sov. Math. 16 (1993), no. 2, 137-150. MR1237836 (94k:57014)

[13] P. B. Kronheimer and T. S. Mrowka, Khovanov homology is an unknot detector, Arxiv.Math/ GT.1005.4346. Publ. Math. Inst. Hautes Études Sci. 113 (2011), 97-208. MR2805599

[14] V. O. Manturov, Parity in knot theory, Mat. Sb. 141 (2010), no. 5, 65-110. Translation: Sb. Math., 201 (2010), no. 5, 693-733. MR2681114 (2011g:57009)

[15] V. O. Manturov, Long virtual knots, Dokl. Akad. Nauk 141 (2005), no. 5, 195-198. Translation: Dokl. Math. 71:2 (2005), 245-248. MR2160133 (2006f:57006)

[16] V. O. Manturov, Knot theory, Moscow-Izhevsk, RCD, 2005. 
[17] V. O. Manturov, Khovanov homology for virtual knots with arbitrary coefficients, Izv. Ross. Akad. Nauk Ser. Mat. 71 (2007), no. 5, 111-148; Translation: Izv. Math. 71 (2007), no. 5, 967-999. MR2362875(2009e:57021)

[18] V. O. Manturov, Parity and cobordism for free knots, to appear in Mat. Sb.

[19] V. O. Manturov and O. V. Manturov, Free knots and groups, Dokl. Akad. Nauk 434 (2010), no. 1, 1-4. Translation: Dokl. Math, 82, no. 2, 697-700. MR2759265

[20] O. V. Manturov and V. O. Manturov, Free knots and groups, J. Knot Theory \& Ramif. 19 (2010), no. 2, 181-186, Arxiv.Math/GT.0912.2694. MR2647053 (2011i:57010)

[21] G. Turaev, Topology of words, Proc. Lond. Math. Soc. (3) 95 (2007), no. 2, 360-412. MR2352565 (2008i:57025)

[22] V. A. Vassiliev, Cohomology of knot spaces, Theory of singularities and its applications, Adv. Sov. Math. 1 (1990), 23-70. MR1089670 (92a:57016)

People's Friendship University, Moscow, Russia

E-mail address: vomaturov@yandex.ru

Translated by G. G. GOULD 